\title{
Bernard COLLETTE-DUčić et Sylvain DELCOMMINETTE (éd.), Unité et origine des vertus dans la philosophie ancienne
}

Laetitia Monteils-Laeng

\section{OpenEdition}

Journals

Édition électronique

URL : https://journals.openedition.org/philosant/468

DOI : 10.4000/philosant.468

ISSN : 2648-2789

Éditeur

Éditions Vrin

Édition imprimée

Date de publication : 24 novembre 2015

Pagination : 276-280

ISBN : 978-2-7574-1141-4

ISSN : $1634-4561$

Référence électronique

Laetitia Monteils-Laeng, "Bernard colletTe-dučić et Sylvain delcomminetTe (éd.), Unité et origine des vertus dans la philosophie ancienne », Philosophie antique [En ligne], 15 | 2015, mis en ligne le 01 novembre 2018, consulté le 03 décembre 2022. URL : http://journals.openedition.org/philosant/468 ; DOI : https://doi.org/10.4000/philosant.468

\section{(c) $(1)$}

Creative Commons - Attribution - Pas d'Utilisation Commerciale - Pas de Modification 4.0 International - CC BY-NC-ND 4.0

https://creativecommons.org/licenses/by-nc-nd/4.0/ 
Elenchos 3 [1982], 55-85), Lampe could have provided a stronger philosophical outlook for some of the views he attributes to Theodorus (and to Hegesias too).

After having surveyed ancient Cyrenaicism, Lampe adds a chapter on the appropriation of Cyrenaicism on the part of Walter Pater (chapter 9: "The New Cyrenaicism of Walter Pater"), thus preferring to deal with the lively influence that Cyrenaicism had on subsequent hedonism than to focus on the ancient debate on the topic of pleasure, namely the debate between Epicureans and Cyrenaics (especially later Cyrenaics). The book ends with a brief conclusion ("Conclusion: the birth of hedonism") and two very instructive appendices, one providing a detailed account of all the main sources on the Cyrenaics and their thought ("Appendix 1: the Sources"), the other discussing a crucial passage in Diogenes Laertius (II 86-93), a passage where Lampe sees an Annicerean interpolation at work (“Appendix 2: Annicerean Interpolation in D.L. 2.86-93”). A lengthy bibliography and an index are also added.

Again, the book deserves to be widely read and is an extremely welcome addition to modern treatments of ancient hedonism. Even when you do not agree with it, Lampe's study works as a stimulus to re-work your own ideas in light of his exhaustive and refreshing handling of Cyrenaic ethics.

Ugo ZILIOLI

Université de Durham

B. Collette-DuČić et S. DelComminetTe (éd.), Unité et origine des vertus dans la philosophie ancienne, Bruxelles, Ousia, 2014 (Cahiers de philosophie ancienne), 478 p. ISBN 978-2-87060-171-6

Ce volume propose de retracer l'histoire des questions relatives à la théorie de l'unité et de l'origine des vertus dans la philosophie ancienne. Réunissant, pour la plupart, les contributions présentées lors d'un colloque organisé à l'Université libre de Bruxelles par le Groupe de philosophie ancienne du Centre de Philosophie, les 24 et 25 mars 2011, l'ouvrage, par l'ampleur de la période qu'il couvre, constitue une entreprise jusque-là inédite. Il propose une lecture qui ne prétend pas à l'exhaustivité, mais qui cherche «à fournir un panorama relativement complet des manières dont cette question a été abordée au cours d'une période déterminée de l'histoire, en couvrant la plupart des épisodes charnières de l'évolution de son traitement » (p. 17).

L'ouvrage s'ouvre sur un article de L.-A. Dorion, « L'introuvable unité des vertus dans les Mémorables de Xénophon » (p. 19-38), qui montre qu'on ne trouve chez le Socrate de Xénophon aucun équivalent des paradoxes dits de l'unité des vertus ou de la vertu-science. Toutes les vertus sont des formes de sophia, mais celle-ci se décline en autant de domaines de compétence susceptibles d'être connus. Il n'y a donc pas d'unité de la vertu chez Xénophon, car il n'y a pas d'unité de la sophia. 
Trois articles sont consacrés à la question telle qu'elle apparaît chez Platon. M.-A. Gavray («Origine et unité de la vertu dans le Protagoras», p. 39-64) restitue la théorie de la vertu de Protagoras. Selon l'auteur, ce n'est qu'artificiellement coupée de son contexte que la question peut sembler socratique. La vertu qu'enseigne le sophiste renvoie à un « code commun» (p. 42) garantissant la cohésion de la cité. Protagoras serait un conformiste, encourageant toutefois chez ses disciples, par des «pratiques réflexives sur l'instrument de l'éducation [les poètes] » (p. 50), une forme d'esprit critique. Le public visé serait non pas la future élite dirigeante, mais l'ensemble des citoyens. Cette lecture minore toutefois la restriction de fait imposée par les conditions d'accès financières à son enseignement assumées par Protagoras (328a-c). Sur la question de l'unité des vertus, Protagoras défend la version lâche d'un tout composé de parties hétérogènes exerçant entre elles des relations de proximité. L'article de D. Sedley, « The unity of virtue after the Protagoras » (p. 65-90), s'inscrit dans la lignée des interprétations dites évolutionnistes des Dialogues. Le modèle intellectualiste développé dans le Protagoras et dans le Phédon est éclipsé par un schéma structuraliste dans le Gorgias et au livre IV de la République. Le Gorgias substitue à la sagesse la modération comprise comme kosmos et non plus comme « state of understanding » (p.74), alors que la République fait de la justice la vertu fondamentale. On peut alors envisager une alternative à la connaissance comme voie d'accès à certaines vertus, tandis que la thèse d'une inséparabilité forte des vertus doit être assouplie. Pour D. Sedley, dans la République (443c9-444a2), c'est la justice qui est la «master-virtue (p. 80) et non la sophia qui lui est néanmoins associée et qu'il comprend comme «practical wisdom $»$ (ibid.). À cette conclusion, on pourrait toutefois objecter que l'ordre avec lequel se confond la justice est lui-même garanti en amont par la possession de la sophia qui préside à son maintien et donc le conditionne. Il peut néanmoins exister des alternatives à la connaissance dans l'accès à la vertu. Ces vertus produites par d'autres voies ne sont cependant pas la vertu complète du philosophe, mais des vertus « imparfaites », à l'image du courage des auxiliaires (R. Kamtekar, «Imperfect Virtue », Oxford Studies in Ancient Philosophy, 18, 1998, p. 315-339), produites par le conditionnement et associées à une opinion droite. A. Giavatto («Unité et articulations de la vertu dans le Politique et dans les Lois de Platon », p. 91-118) revient sur ce qui semble être une anomalie chez Platon: la these incompatibiliste selon laquelle courage et modération seraient des vertus ennemies (Politique, 306a-308b; 308b-311c). L'«amplification conceptuelle » (p. 101) dont font ici l'objet les rapports contrariés de ces deux vertus s'expliquerait par la volonté d'insister sur la capacité de conciliation qui doit être celle du politique.

Les deux articles suivants sont consacrés à Aristote. S. Delcomminette («Unité des vertus et unité du bien chez Aristote », p. 119-143) tente de réconcilier phronesis et sophia, en montrant notamment que la visée ultime de l'existence bonne est la contemplation, tandis que la phronesis représente la condition de pos- 
sibilité de la sophia (p. 139). Ce faisant, l'auteur défend l'idée d'une unité « architectonique » (p. 142) des vertus, allant même jusqu’à rassembler, en un sens, vertus éthiques et vertus dianoétiques. D. Lefebvre («Séparation, dépendance et unité des vertus chez Aristote et quelques péripatéticiens », p. 146-211) adopte une perspective inverse de celle de S. Delcomminette : loin d'affirmer l'unité absolue des vertus, le Stagirite interrogerait plutôt la possibilité de leur pluralité tout en maintenant une forme de dépendance entre elles. Le statut prédominant accordé à la prudence vient en effet complexifier le problème, en introduisant une forme d'asymétrie entre celle-ci et les vertus éthiques. L'auteur révèle une première tension entre une unité de définition qui englobe les vertus éthiques et la propension qu'a le philosophe à restreindre chaque vertu à un domaine particulier de l'action, ce qui pourrait mettre en péril non seulement une éventuelle unité des vertus mais aussi la possibilité même d'un dénombrement exhaustif. Est abordée ensuite la double difficulté du rapport des vertus éthiques à la prudence et aux vertus naturelles dont la temporalité particulière - elles apparaissent à différents âges de la vie - remet en cause l'idée que l'on puisse posséder toutes les vertus en même temps. L'auteur montre qu'à la fin du livre VI de l'EN, il s'agit surtout d'insister sur le rôle unifiant de la prudence à l'endroit des vertus éthiques et non de souligner « une relation de réciprocité entre des vertus éthiques » (p. 176). La seconde difficulté se résout dès lors que l'apparition successive des vertus naturelles ne conduit pas à une forme de conflit entre vertus ou à la cohabitation de certaines vertus avec des vices (p. 166-167). La deuxième partie de l'article est consacrée à la reprise péripatéticienne de la question et mesure l'influence exercée sur celle-ci par le stoïcisme, notamment au travers de la notion d'antakolouthia (entre-implication) étrangère à la terminologie aristotélicienne. Les différentes interprétations proposées par Théophraste (implication réciproque mais dissymétrique entre les vertus éthiques et la prudence), Aspasius (implication réciproque partielle et restreinte à certaines vertus), et Alexandre d'Aphrodise (implication réciproque associée à une inséparabilité des vertus) révèlent les incertitudes qui subsistent quant à la position aristotélicienne sur la question, mais aussi la fécondité de leur reformulation dans une terminologie stoïcienne (p. 210).

J. Warren («Epicurus and the unity of the virtues », p. 214-236) montre que le Jardin a participé pleinement au débat : l'identification du souverain bien au plaisir n'interdit pas que la vertu occupe une place de premier ordre au sein de la vie bonne (vertu et vie agréable sont inséparables et co-dépendantes, p. 220); l'unité des vertus est assurée dès lors qu'elles découlent naturellement de la phronesis.

Deux articles traitent de la question au sein du Portique. B. Collette (« L'unité des vertus chez Zénon de Citium et son interprétation chrysippéenne », p. 237 267) part d'un témoignage partiellement corrompu de Plutarque (Contradictions stoïciennes, $7,1034 \mathrm{C}$ ) sur Zénon. Plutarque prétend que Zénon se contredit en affirmant l'existence d'une pluralité de vertus et en les réduisant à différentes ex- 
pressions de la phronesis. Contre cette lecture, l'auteur propose de distinguer deux emplois de phronesis, comme vertu générique et comme vertu spécifique au côté des vertus cardinales, selon « une dénomination par éponymie (où le tout donne son nom à une partie » (p. 249). Hypothèse corroborée par Chrysippe qui substitue à la phronesis l'episteme, faisant de chaque vertu une espèce de science, et par Cléanthe pour qui l'enkrateia joue un rôle similaire à la phronesis zénonienne. J.-B. Gourinat («Hétérodoxies stoïciennes sur l'unité des vertus », p. 269-296) discute d'abord de la légitimité d'une quelconque orthodoxie stoïcienne sur la question de l'unité des vertus, mise à mal par un témoignage de Diogène Laërce (VII 92). Reste que, mis à part la conception hétérodoxe d'Ariston, les supposées hétérodoxies de Panétius (vertus théoriques et vertus pratiques) et de son disciple Hécaton (vertus théoriques et vertus athéoriques) doivent être nuancées. Chez le premier, la spécificité de la prudence ne met cependant pas en péril l'entrelacement de toutes les vertus (Cicéron, De Officiis, I 15); tandis que, pour le second, l'introduction d'une implication non réciproque (les vertus athéoriques dépendent des théoriques, mais l'inverse n'est pas vrai) n'est « pas en soi incompatible avec la théorie standard de la vertu comme science $\gg$ (p. 290).

Trois études sont consacrées au platonisme et au néo-platonisme. G. BoysStones («Unity and the Good: Platonists against oikei $\omega \sigma \iota \varsigma »$, p. 297-320) restitue le débat qui a opposé stoïciens et péripatéticiens aux platoniciens post-hellé-

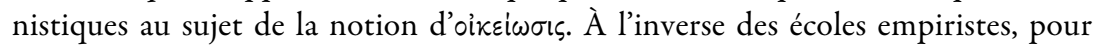
qui la moralité coïncide avec une forme raffinée des impulsions qui guident les ani-

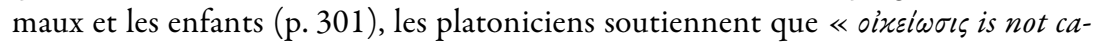
pable of underwriting a well-founded sense of objective value » (p. 310). Le mécanisme de l'appropriation à soi peut certes initier une conduite en vue d'une fin, mais ne saurait fonder une notion stable et objective du bien. A. Schniewind («Plotin et les émotions nobles», p. 321-337) propose d'éclairer les rapports entre vertus civiles et vertus supérieures en analysant les «émotions nobles » qu'éprouve le sage plotinien, qu'elle rapporte aux «émotions éclairées » de MarcAurèle. L'apathie stoïcienne implique de se débarrasser des émotions viles toujours susceptibles de provoquer des épisodes d'akrasia que l'auteur rend de façon un peu anachronique par «faiblesse de volonté » (p. 327). L'akrasia est, pour les stö̈ciens, paradigmatique de la passion qui nous emporte (Galien, PHP, IV 4, 24-25, p. 256 De Lacy) et ne suppose en aucune façon un manque de volonté. Cet idéal n'interdit toutefois pas de ressentir de la joie. On pourrait toutefois rappeler que ces émotions accessibles au sage apparaissent dès l'ancienne Stoa sous l'appellation eupatheiai (DL, VII 116). D. Cohen («L'unité des vertus dans le néoplatonisme tardif », p. 339-362) explore les hiérarchies des degrés de vertus mises en place par Porphyre (quatre degrés) et par Jamblique, Proclus, Hiéroclès, Damascius et Olympiodore (sept degrés). Ces auteurs revisitent le modèle de l'antakolouthia des vertus en empruntant des outils conceptuels (p. 346) au stoïcisme (théorie du mélange total) et à Anaxagore (nature et appellation d'une réalité déterminées par ce 
qui prédomine dans un mélange), pour penser la multiplicité des degrés selon un schéma de continuité analogique (p. 343).

O. Gilon («Vertus cardinales et théologales chez saint Augustin », p. 363388) revient sur le double héritage - hellénistique et paulinien - du saint Docteur. Les vertus ne peuvent se réaliser sans le secours de la grâce divine. L'indépendance de la volonté par rapport à l'intelligence signifie que la connaissance ne suffit pas à fonder la vertu (p. 373). Augustin s'écarte encore de la philosophie ancienne en admettant des étapes intermédiaires entre l'absence de sagesse et son acquisition, mais aussi en niant que la pleine possession de la vertu soit accessible ici-bas.

Le volume se clôt sur un article de M. Dixsaut, «Deux éthiques aristocratiques » (p.389-417), qui rapproche deux perspectives traditionnellement opposées, celles de Platon et de Nietzsche qui partagent une « conception aristocratique de la vertu » (p.416) : le projet de perfectionner l'humain par la promotion d'un certain « type » d'homme, la dénonciation de la falsification des valeurs, l'invention d'une nouvelle culture/éducation sont autant de thèmes autour desquels se retrouvent les deux penseurs.

L'ouvrage comprend une conclusion (p.419-434), une riche bibliographie (p. 435-452), un index locorum (p. 453-471) et un index nominum des auteurs modernes (p. 473-476). Les quatorze études réunies ici proposent des approches détaillées et souvent originales sur les différents traitements qu'a pu recevoir la question. On pourrait toutefois s'étonner de ce qu'aucune ne soit consacrée à l'aspect socratique et aux dialogues (Protagoras, Ménon) qui l'initient. La conclusion propose néanmoins quelques pistes encore à explorer sur ce sujet (p. 419-420), prouvant que, bien qu'abondamment discutée, la question de l'unité des vertus demeure un champ d'investigation stimulant.

Laetitia MONTEILS-LAENG

Université de Montréal

Roberto Polito (éd.), Aenesidemus of Cnossus. Testimonia, Cambridge, Cambridge University Press, 2014 (Cambridge Classical Texts and Commentaries, 52), ISBN 9780521190251.

L'importance d'une édition des témoignages d'Énésidème s'impose de toute évidence pour tous ceux qui s'occupent du scepticisme grec. Le problème est bien connu: le pyrrhonisme constitue un des mouvements philosophiques les plus intéressants de l'Antiquité et son influence a traversé les siècles jusqu'aujourd'hui. Mais qu'est-ce que le pyrrhonisme, quels sont ses origines et son développement ? Nous sommes informés sur les deux extrêmes de cette histoire, Pyrrhon et Sextus Empiricus. Mais Pyrrhon n'était pas pyrrhonien: son indifférentisme n'a pas beaucoup à faire avec la philosophie du scepticisme; Sextus Empiricus, le chapitre conclusif du pyrrhonisme antique, est en revanche un sceptique avéré, mais il semble être plutôt un rapporteur qu'un penseur original. L'édition de R. Polito nous permet enfin de concentrer notre attention sur le penseur qui réside au 